\title{
Single Molecular and Sub-Zeptoliter Thermal Probing
}

\author{
R. Proksch, A. Moshar
}

Asylum Research, 6310 Hollister Ave. Santa Barbara, CA 93117

Conventional local thermal analysis (LTA) uses the probe deflection as a function of the tip temperature to measure localized thermal expansion. This scanning probe microscopy technique has been used for many years to examine changes in bulk properties of a variety of samples in response to heating. A standing problem with existing atomic force microscopy-based thermal analysis systems is thermally induced bending of the cantilever that results in spurious deflection signals and variable loads being applied during heating. Using a relatively new Atomic Force Microscope technique dubbed Dual AC Resonance Tracking (DART) provides much higher sensitivity to thermomechanical changes at tip-sample junction and also allows the cantilever deflection to be used in a feedback loop, stabilizing the position and minimizing sample damage. It corrects the above-mentioned problem, providing constant-load detection of thermally induced melting (Tm), phase transitions (Tg) and other morphological and compliance effects for materials studies and identification for areas less than $20 \mathrm{~nm}$ x $20 \mathrm{~nm}$. In addition to tracking the resonance frequency, this technique allows the tip-sample dissipation to be measured, a quantity that shows very strong temperature dependence in a wide variety of samples, including polymers,

Contact resonance and dissipation measurements on isolated single biomolecules such as DNA, collagen, IgG and insulin will be presented. The measurements show diverse and repeatable features up to the point of decomposition. In these cases, the volume of material probed is on the order of 100-500 yocto-liters. The measurements will be discussed in the context of bulk thermal measurements of protein denaturing. In addition, thermal measurements of polymer films including PETE and SEBS show measureable and repeatable thermal behavior. AFM images made before and after the measurements show that the resonance measurements have a sensitivity beyond what can be reliably differentiated with the AFM - in other words, the sample roughness is larger than the scale of the features irreversibly transformed in the thermal measurement. As with the isolated single molecular measurements, the volume of irreversibly affected material is estimated to be in the sub-zeptoliter range. 